\title{
The use of classification tree analysis to assess the influence of surgical timing on neurological recovery following severe cervical traumatic spinal cord injury
}

\author{
Yann Facchinello ${ }^{1,2} \cdot$ Andréane Richard-Denis $^{2,3} \cdot$ Marie Beauséjour ${ }^{1,4} \cdot$ Cynthia Thompson $^{2} \cdot$ \\ Jean-Marc Mac-Thiong ${ }^{1,2,4}$
}

Received: 15 October 2017 / Revised: 9 January 2018 / Accepted: 29 January 2018 / Published online: 26 February 2018

(c) International Spinal Cord Society 2018

\begin{abstract}
Study design Post hoc analysis of prospectively collected data.

Objectives Assess the influence of surgical timing on neurological recovery using classification tree analysis in patients sustaining cervical traumatic spinal cord injury.

Setting Hôpital du Sacré-Coeur de Montreal

Methods 42 patients sustaining cervical SCI were followed for at least 6 months post injury. Neurological status was assessed from the American Spinal Injury Association impairment scale (AIS) and neurological level of injury (NLI) at admission and at follow-up. Age, surgical timing, AIS grade at admission and energy of injury were the four input parameters. Neurological recovery was quantified by the occurrence of improvement by at least one AIS grade, at least 2 AIS grades and at least 2 NLI.

Results Proportion of patients that improved at least one ASIA grade was higher in the group that received early surgery (75 vs. $41 \%)$. The proportion of patients that improved two AIS grades was also higher in the group that received early surgery (67 vs. $38 \%$ ). Finally, $30 \%$ of the patients that received early decompression improved two NLI as compared with $0 \%$ in the other group. Early surgery was also associated with a non-statistically significant improvement in functional recovery. Conclusions Neurological recovery of patients sustaining cervical traumatic spinal cord injury can be improved by early decompression surgery performed within $19 \mathrm{~h}$ post trauma.
\end{abstract}

Sponsorship U.S. Army Medical Research and Material Command, Rick Hansen Institute.

\section{Introduction}

Following traumatic spinal cord injuries (TSCI), motor, and sensory functions can be severely impaired, leading to a loss

Jean-Marc Mac-Thiong

macthiong@gmail.com

1 Department of Surgery, Faculty of Medicine, University of Montreal, Pavillon Roger-Gaudry, S-749, C.P. 6128, succ. Centreville, Montreal, Quebec H3C 3J7, Canada

2 Hôpital du Sacré-Cœur de Montréal, 5400 Gouin Boul. West, Montreal, Quebec H4J 1C5, Canada

3 Department of Medicine, Faculty of Medicine, University of Montreal, Pavillon Roger-Gaudry, S-749, C.P. 6128, succ. Centreville, Montreal, Quebec H3C 3J7, Canada

4 Sainte-Justine University Hospital Research Center, 3175 Chemin de la Côte-Sainte-Catherine, Montréal, Quebec H3T 1C5, Canada of autonomy and a poor quality of life. The incidence of TSCI ranges from 10 to 80 cases per million inhabitants depending on the country, whereas the prevalence varies from 250 to 950 cases per millions [1].

Surgical intervention is generally required following TSCI, in order to stabilize the spine, relieve the mechanical pressure to the spinal cord, and potentially minimize the cascade of secondary lesions to the spinal cord.

Following TSCI, neurological recovery is one of the main concerns for patients as it is directly related to their independence, quality of life, and productivity [2]. Number of demographical and clinical parameters influence neurological recovery, such as the injury severity, age, occurrence of medical complications, injury mechanism, and energy [ 3 , 4]. Among those parameters, surgical delay, defined as the time interval between the trauma and surgical intervention, has a significant role in the long-term functional and neurological recovery [5]. Indeed, shorter surgical delays were 
Fig. 1 Flow chart of patients excluded from the study

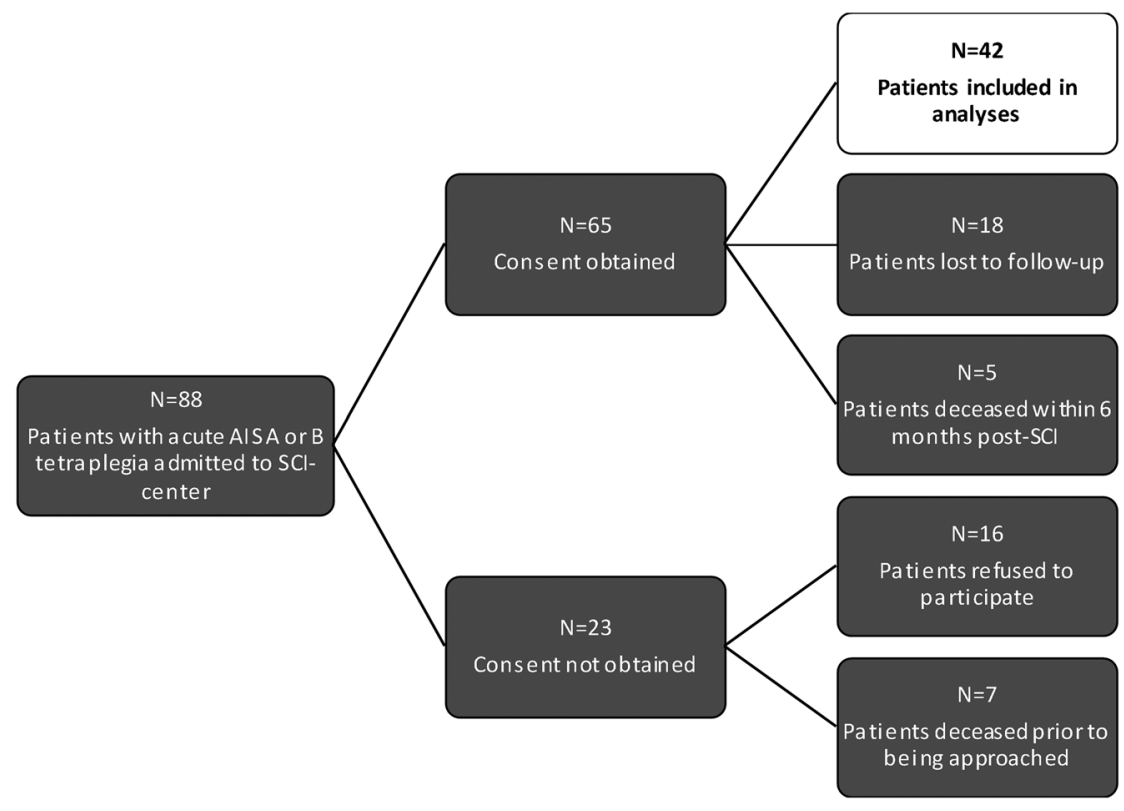

showed to lead to better outcomes, less complications, and decreased resource utilization but the level of evidence regarding those observations is considered as 'low' according to the recently published AOSpine guidelines [6]. The definition of early surgery is still debatable as values ranging from 8 to $72 \mathrm{~h}$ can be found in the literature [6, 7]. It also must be noted that the definitions of early surgery found in the literature are arbitrary and no attempt was made to define an optimized surgical timing, leading to better neurological outcome. Patients sustaining acute tetraplegia may particularly benefit from early surgery, especially in terms of improved neurological recovery as recently shown by Bourassa-Moreau et al. [8] and Fehlings et al. [5].

The surgical delay is highly variable as it is influenced by several factors such as the duration of the transfer from the trauma location to the acute care center, operating room availability, comorbidities, and surgeon availability [9]. Although the impact of surgical timing on several outcomes is being highly studied as it is one of the only modifiable parameters during the acute care period after TSCI [9], the optimal surgical timing associated to the greatest recovery is still unknown. Previous works have arbitrary defined early decompression as a surgical intervention performed within $24 \mathrm{~h}$ post trauma, although a $72 \mathrm{~h}$ cutoff has also been used [6]. The concept of ultra-early decompression, defined as a surgical intervention performed within $8 \mathrm{~h}$ post injury, was also recently introduced by Burke et al. [7] as a way to improve the recovery following TSCI. Currently, threshold values to define early surgery remain empirical and arbitrary, as previous authors have never attempted to determine threshold values based on objective analysis.
The main objective of this study is to assess the influence of surgical timing of the neurological recovery following TSCI in patients sustaining motor-complete cervical TSCI and to determine a specific surgical delay leading to better neurological recovery using classification tree analysis. This statistical method was used for the first time to assess the influence of surgical timing on the neurological recovery following TSCI. Classification tree analysis repeatedly partitions a data set into two subgroups based on an objective splitting criterion (predictor most related to the outcome) until further partitioning no longer adds value to the prediction. Therefore, our study relies on the objective identification of a splitting criterion at each level of a classification tree for maximizing the discrepancy between the generated subgroups, in order to identify the optimal timing of surgery associated with highest likelihood of neurological recovery.

\section{Methods}

\section{Participants}

This study was based on a prospective cohort of 42 patients who sustained a motor-complete, cervical TSCI between January 2010 and June 2016. All patients were enrolled on a voluntary basis and signed the informed consent during the acute hospitalization at a single Level I trauma center specialized in TSCI. Patients were included if they sustained a TSCI between C1 and T1 levels with an initial American Spinal Injury Association Impairment Scale (AIS) grade at admission of $\mathrm{A}$ or $\mathrm{B}$ and were followed for a minimum of 
6 months after the trauma. Figure 1 shows the flow chart of patients excluded from the study. This study was approved by the Institutional Review Board. All applicable institutional and governmental regulations concerning the ethical use of human volunteers were followed during the course of this research.

\section{Variables}

\section{Outcome variables}

The International Standards for Neurological Classification of Spinal Cord Injury were used to assess the severity of injury in the form of AIS grades at 6 months follow-up minimum for every patient [10]. The third version of the Spinal Cord Independence Measure was used to quantify the functional outcome at 6-month follow-up. Improvement by at least one AIS grade was considered as the primary endpoint to assess neurological recovery. An improvement by at least two AIS grade [11] and by at least two neurological levels of injury (NLI) improvement were considered as secondary outcomes.

\section{Predictor variables}

Parameters influencing the neurological recovery consisted in a reduced number of four independent variables recognized as potential predictors of recovery following TSCI [3, 4]. Age was considered as a continuous variable. Timing of surgery was defined as the interval of time between the trauma and beginning of surgery and was also considered as a continuous variable. The energy of the trauma was considered as low (trivial trauma, fall from standing or walking, assault, etc) or high (motor-vehicle/motorcycle accident, pedestrian hit by vehicle, fall from more than $10 \mathrm{ft}$, etc.). Finally, the initial AIS grade was used to assess the severity of injury for every patient at admission.

\section{Statistical analysis}

Statistical analyses were performed using the classification and regression tree (CART) analysis engine of the Salford Predictive Modeler software (Version 8, Salford Systems, San Diego, CA, USA). The classification trees were constructed using the Gini splitting rule. A stopping rule was used to prevent the algorithm from creating subgroups of five patients or less. Overfitting was monitored by choosing the tree exhibiting the minimum relative cost value computed by the software. Trees were then pruned to prevent splitting rules based on the surgical timing from appearing more than once.

The relative importance of each predictor was computed for the three dependent variables (one AIS grade improvement; two or more AIS grade improvement; two or more NLI improvement). Variable importance reflects the relative influence of each predictor on the endpoint. The most important predictor during tree construction was assigned with a score of 100 and the other predictors were scaled down proportionally to their importance. The classification and regression tree analysis engine of the Salford Predictive Modeler software identifies surrogate splitter, as close approximations of the primary splitters appearing in the trees [12]. Surrogates splitter are used by the algorithm to handle eventual missing data and are taken into account when computing the variable importance.

Continuous data were reported as median and interquartile range (IQR), whereas categorical data were reported as percentages. $\chi^{2}$-square tests were used to assess whether the proportion of patients who improved neurologically was statistically different from the proportion of patients who did not improve as determined following the split based on the surgical timing. Mann-Whitney $U$-tests were used to compare mean SCIM and ASIA motor score of subgroups separated by the surgical timing split. The significance level was set at $p<0.05$.

\section{Results}

Table 1 presents the distribution of the four independent variables (input parameters) and three endpoints across the population considered in this study. One or more AIS grade improvement was observed in $57 \%$ of patients, whereas $40 \%$ of patients improved by 2 AIS grades or more. Neurological level of injury improved by at least two levels in $17 \%$ of patients at follow-up. The median age (IQR) at admission was 43 (30-60) years old and the median surgical timing was $19 \mathrm{~h}$ (13-28h). High velocity traumas occurred in $30 \%$ of the cases. AIS grade at admission was A in $71 \%$ of the cases and B in the remaining $29 \%$. The most frequent

Table 1 Input parameters and outcomes considered in this study

\begin{tabular}{lll}
\hline Outcome measures & & \\
\hline 1 or more AIS Grade improvement & & $57 \%$ \\
2 or more AIS Grades improvement & & $40 \%$ \\
2 or more NLI improvement & & $17 \%$ \\
Input parameters & Median (IQR) & $43(30-61)$ \\
Age (years) & Median (IQR) & $19(13-28)$ \\
Delay from injury to surgical incision & & \\
(hours) & High & $30 \%$ \\
Energy associated with the injury & Low & $70 \%$ \\
& AIS A & $71 \%$ \\
ASIA Impairment Scale (AIS) & AIS B & $29 \%$ \\
\end{tabular}


surgical delay ranges from 15 to $20 \mathrm{~h}$ in our cohort, as shown in Fig. 1.

Figure 2 shows the distribution of the surgical timing across the population enrolled in the study. The most frequent timing observed ranges between 15 and $20 \mathrm{~h}$.

Figure 3 presents the classification tree obtained considering one AIS grade improvement as the dependent variable. $57 \%$ of the complete data set showed improvement of at least one AIS grade. This group was splitted based on the surgical delay with a cutoff value of $19 \mathrm{~h} .75 \%$ of the patients that had surgery within $18.9 \mathrm{~h}$ post trauma improved by at least one AIS grade, forming the first terminal node. On the other hand, only $41 \%$ of the patients that had surgery later than $18.9 \mathrm{~h}$ post trauma improved by at least one AIS grade. This group was then divided based on the AIS grade at admission, where $67 \%$ of AIS B patients improved at least one AIS grade, as compared with $23 \%$ of AIS A patients. The surgical timing split the complete data set into two groups showing significantly different proportions of patient that improved at least one AIS grade according to $\chi 2$-test $(p=0.026)$.

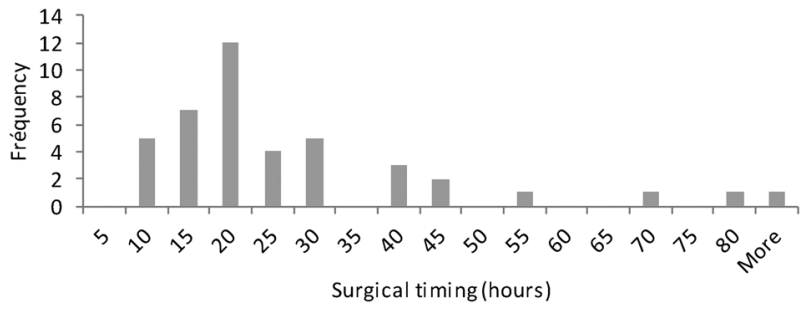

Fig. 2 Distribution of surgical timing across the cohort
The surgical delay was found to be the parameter influencing the most the probability of AIS grade improvement, followed in order of importance by the initial AIS grade, energy of injury and age (as showed as the "importance of variable" box below).

Figure 4 shows the classification tree obtained considering a neurological improvement of at least 2 AIS grades as the dependant variable. $40 \%$ of the complete data set of 42 patients improved 2 AIS grades or more. This group was divided based on the energy associated with the injury. Patients sustaining a high energy injury formed a terminal node in which $14 \%$ of them improved 2 AIS grades of more. A total of $54 \%$ of the patients sustaining a low energy injury improved 2 AIS grades or more. The group of patients associated with low energy injury was then split based on the surgical timing, where $67 \%$ of patients who had surgery within $18 \mathrm{~h}$ post trauma improved by at least two AIS grades. This proportion dropped to $38 \%$ for surgical timings longer than $18 \mathrm{~h}$. The subgroups created following the split based on the surgical timing were significantly different $(p=0.038)$. Energy of injury was the most important variable followed by surgical timing and age. The AIS grade at admission had no influence on whether patients improved by 2 AIS grades or more.

Figure 5 shows the influence of the surgical timing on the improvement of at least two NLI. A total of $17 \%$ of the complete data set showed improvement of two NLI at follow-up. A total of 30\% of patients that had surgery within $20 \mathrm{~h}$ post trauma gained at least two NLI, whereas none that had surgery later than $20 \mathrm{~h}$ post trauma improved by at least two NLI. The two subgroups created following the split were significantly different $(p=0.016)$. Surgical

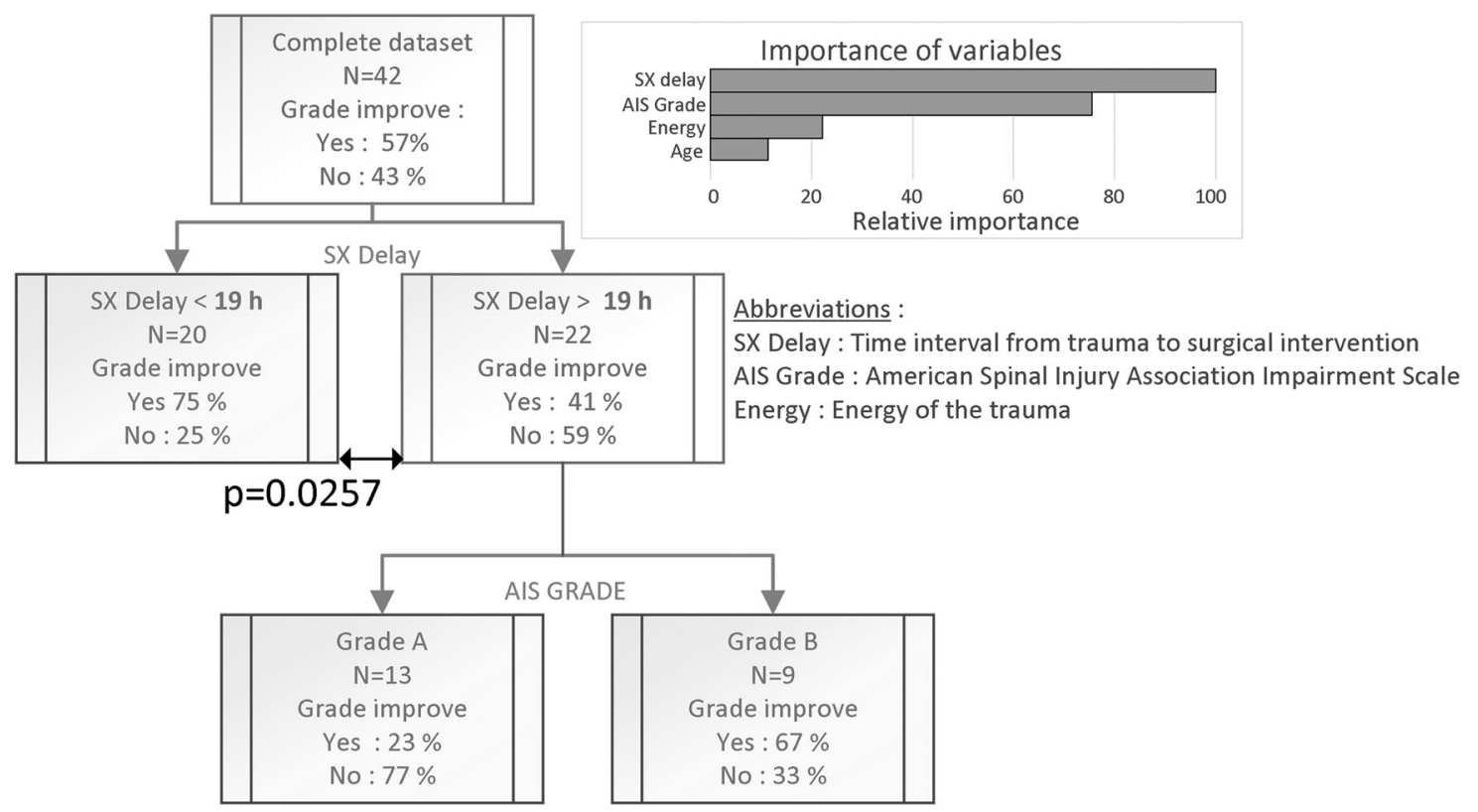

Fig. 3 Classification tree describing the influence of the 4 parameters under study on the one AIS grade neurological improvement 


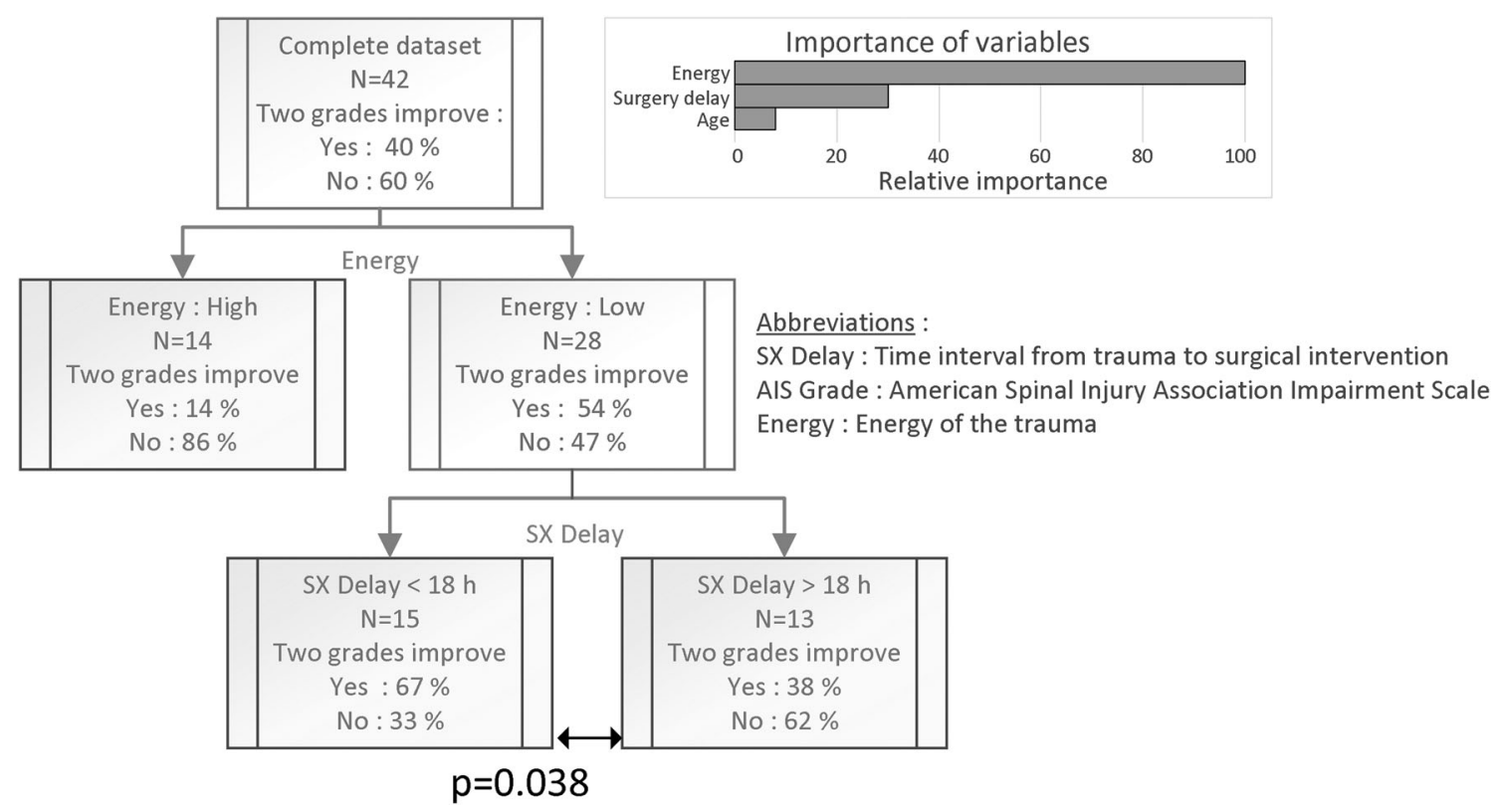

Fig. 4 Classification tree describing the influence of the 4 parameters under study on at least 2 AIS grades neurological improvement

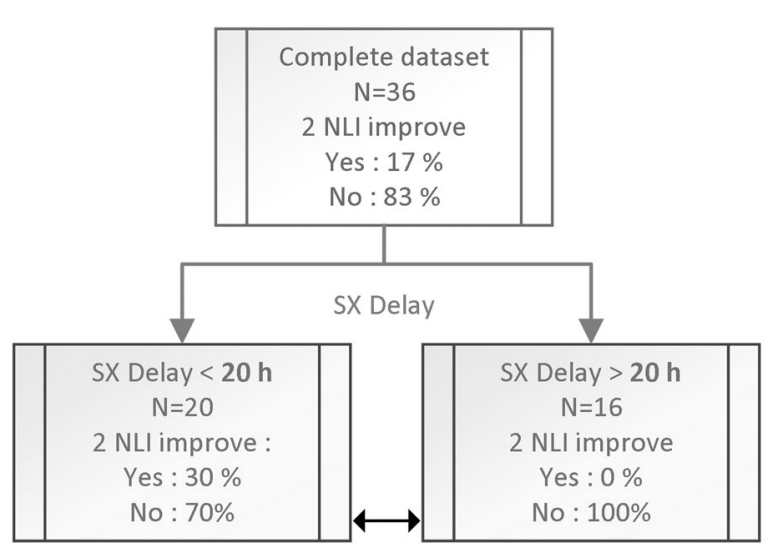

$p=0.0164$

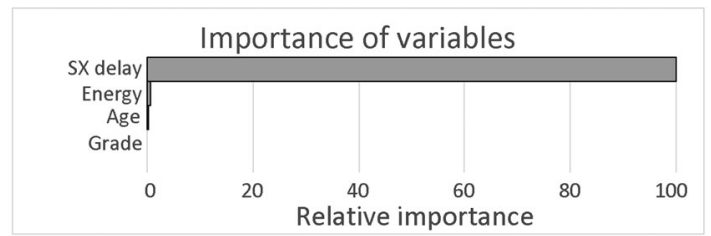

Abbreviations :

SX Delay : Time interval from trauma to surgical intervention AIS Grade : American Spinal Injury Association Impairment Scale Energy : Energy of the trauma

NLI : Neurological level of injury

Fig. 5 Classification tree describing the influence of the 4 parameters under study on the gain of at least two neurological level of injury

timing was the most important parameter in this tree. The other parameters had almost no influence on the proportion of patients gaining two NLI or more.
Table 2 shows the mean SCIM and ASIA motor scores at follow-up of subgroups created by the $19 \mathrm{~h}$ surgical timing split. The table also shows the $p$ values associated with the Mann-Whitney $U$-tests used to assess the significance of the difference between both subgroups. Decompression surgery performed within $19 \mathrm{~h}$ post injury increased the mean SCIM and ASIA motor score by 7 and 3.7 points respectively. The improvements in SCIM and ASIA motor scores were not statistically significant according to Mann-Whitney tests ( $p$ values of 0.4902 and 0.6312 , respectively)

\section{Discussion}

This study proposes the use of classification tree algorithms to assess the effect of surgical timing on neurological recovery and to justify a threshold value defining the concept of early surgical intervention frequently described in the literature. This study is the first to determine specifically a surgical timing leading to better outcomes for a particular subgroup of patients

The delay prior to surgery influenced significantly neurological recovery in terms of AIS grade and NLI improvement. According to the results obtained in this study, a surgical intervention within $19 \mathrm{~h}$ post trauma improves the likelihood of neurological recovery for patients sustaining complete motor TSCI at the cervical level. A cutoff value approximating $19 \mathrm{~h}$ was observed for all three outcomes, confirming the relevance of this observation. Our 19-hour surgical delay is in good agreement 
Table 2 SCIM and ASIA motor scores of subgroups created by the $19 \mathrm{~h}$ surgical timing split and $p$ values associated with the Mann-Whitney tests

\begin{tabular}{llll}
\hline & Timing $<19 \mathrm{~h}$ & Timing $>19 \mathrm{~h}$ & Mann-Whitney $U$-test $p$ value \\
\hline SCIM score: Mean (SD) & $46(29,4)$ & $39,4(26,2)$ & 0,4902 \\
ASIA motor score: Mean (SD) & $40,8(29)$ & $37,1(31,1)$ & 0,6312 \\
\hline
\end{tabular}

with previously published studies showing that an early surgical intervention improves the neurological recovery [5, 13-15]. For instance, Umerani et al. (2014) [15] reported a mean surgical delay of $18 \mathrm{~h}$, which was associated to a greater proportion of patients improving by at least 2 AIS grades as compared with patients operated on average $53 \mathrm{~h}$ post SCI. These results are also similar to what was shown by Fehlings et al. (2012) [5], where tetraplegic patients operated on average $14 \mathrm{~h}$ post SCI improved of at least 2 AIS grades in a greater proportion than who had later surgery. The value of $19 \mathrm{~h}$ is also clinically relevant as it is a realistic target for health care workers considering all the factors contributing to a longer delay prior to surgery [9].

Decompression surgery performed within $19 \mathrm{~h}$ post injury increased the mean SCIM score by seven points, which can be considered as an average meaningful improvement according to the study published by Scivoletto et al. [16]. Mean ASIA motor score increased by 3.7 points which can be described as a very small improvement according to Scivoletto et al. [17]. Both improvements were not statistically significant according to the Mann-Whitney $U$-tests.

The results indicate that the influence of surgical timing may be more important for neurological recovery than for functional recovery. This is in accordance with the literature since early surgery is consistently associated with improved neurological outcome [5, 8], while the association between early surgery and improved function is more limited. More specifically, Grassner at al. [18], found that early surgery was associated with better functional outcome whereas other studies showed that surgical timing had no significant influence on functional recovery [3, 4].

The pathophysiology of human TSCI is poorly understood. However, several animal studies showed that shorter compression duration can lead to better neurological recovery. In a meta-analysis, El Tecle et al. [19] found that all pre-clinical studies published after 1 January 2000 were all in favor of early decompression, but those studies were heterogeneous regarding the definition of early surgery. The same observation was made by Batchelor et al. [20]. Various pre-clinical studies also showed that increasing compression durations are associated with more severe tissue destruction and sometime declining behavioural measure [21-23]. More specifically, Shields et al. [24] found that the lesion volume increased significantly with the increasing compression duration from 12 to $24 \mathrm{~h}$ in a rodent spinal cord injury model subjected to spinal cord contusion immediately followed by $43 \%$ spinal canal stenosis. This observation is directly in line with the results presented in our study suggesting that a major degradation might occur for spinal cord compression duration ranging from 12 to 24 h.

The optimal surgical timing varied slightly from 18 to 20 $\mathrm{h}$ post injury depending on the outcome measure considered. This observation shows that the optimal surgical timing depends on the outcome considered which might explain the large spread of early surgical timing values found in the literature $[19,25]$. The optimal surgical timing must therefore be defined considering the severity of the SCI (complete or incomplete SCI), the neurological outcome considered, as well as the neurological level of injury.

The analysis presented in this communication considered age, AIS grade at admission, energy of the trauma and surgical timing as parameters potentially influencing the neurological recovery. A recent systematic review identifying factors associated to neurological outcome, Yousefifard et al. (2017) [26] reported that many clinical, neurological, and demographic characteristics can be used to assess the potential of recovery. Among the most common, younger age, less severe AIS grades at admission as well as lower velocity of trauma are considered by several studies among the most important factors influencing the neurological recovery following TSCI. In our study, the surgical timing was found to be one of the most important parameter affecting the three outcome measures, showing that the neurological recovery can be strongly influenced by one of the only modifiable parameters in the acute treatment of TSCI.

It is worth noting that the proportion of patients who improved by one or at least two AIS grades, respectively, $57 \%$ and $40 \%$, might seem high as compared with previously published data [5, 27, 28] Several hypotheses can be raised to explain this result. Patients with sensory incomplete tetraplegia (AIS B) have a better prognosis for neurological recovery [18]. Although the majority of our cohort had a complete AIS A and that this factor did not come out as a predictor in the CART analyses, it cannot be ruled out. Kirshblum SC et al. (1998) [29] also reported that most patients who had an AIS A injury, as assessed within $72 \mathrm{~h}$ of trauma, and who improved by more than one AIS grade also sustained head injuries involving cognitive impairments, and were thus incorrectly diagnosed as AIS A. 
This could also have occurred in the present study, where the initial AIS grade considered were mainly obtained within $72 \mathrm{~h}$ post SCI [29], which could have lead to an overestimation of the severity of the injury.

\section{Study limitations and recommendations}

As shown in Fig. 1, most patients had surgery between 15 and $20 \mathrm{~h}$ after the TSCI, with a median of $19 \mathrm{~h}$. However, the cohort under study still exhibited a large spread of surgical timing ranging from 8 to $250 \mathrm{~h}$ post trauma, which could have allowed identification of an optimal timing of surgery outside the $15-20 \mathrm{~h}$ range.

This study was performed on a limited number of patients, and further study with a larger sample should be done to reinforce our conclusion. However, our sample size was sufficient to reach our objectives and observe the significant influence of the timing of surgery on our endpoints.

The results presented in this post hoc analysis of prospectively collected data suggest that decompression surgery performed within $19 \mathrm{~h}$ post trauma could help optimizing the neurological recovery following TSCI. However, this association may be related to biases in the cohort rather than a direct causative relationship. In order to confirm this observation, prospective studies involving a larger cohort should be performed with all possible predictors and confounders identified through directed acyclic graphs as suggested by Shrier and Platt [30].

Finally, including neurological levels from $\mathrm{C} 1$ to $\mathrm{T} 1$ might lead to a ceiling effect when considering improvements of two neurological levels or more, as it is difficult to assess motor improvement at levels below $\mathrm{T} 1$.

\section{Conclusion}

Surgical timing following TSCI has been thoroughly studied as it is related to a main treatment strategy (spine surgery) during the acute care of TSCI, and represents one of the infrequent modifiable parameters of the acute care hospitalization. The use of classification tree analysis was proposed to assess the influence of surgical timing on the neurological recovery in patients sustaining cervical motorcomplete TSCI and to justify an optimal surgical timing leading to better neurological outcome. A surgical intervention performed within $19 \mathrm{~h}$ post injury was associated with significant improvement in neurological recovery. This study is the first to define a surgical timing value optimized for a specific subgroup of patients. The methodology described in this communication could be applied to a larger cohort involving patients with different severity and levels of TSCI.
Acknowledgements This work was supported by the US Army Medical Research and Material Command and by the Rick Hansen Institute.

\section{Compliance with ethical standards}

Conflict of interest The authors declare that they have no conflict of interest.

\section{References}

1. Singh A, Tetreault L, Kalsi-Ryan S, Nouri A, Fehlings MG. Global prevalence and incidence of traumatic spinal cord injury. Clin Epidemiol. 2014;6:309-11.

2. Steeves JD, Lammertse D, Curt A, Fawcett JW, Tuszynski MH, Ditunno JF, et al. Guidelines for the conduct of clinical trials for spinal cord Guidelines injury ( $\mathrm{SCI}$ ) as developed by the ICCP panel: clinical trial outcome measures. Spinal Cord. 2007;45:206-221.

3. Facchinello Y, Beauséjour M, Richard-Denis A, Thompson C, Mac-Thiong J-M. The use of regression tree analysis for predicting the functional outcome following traumatic spinal cord injury. J Neurotrauma. https://doi.org/10.1089/neu.2017.5321 2017.

4. Richard-Denis A, Feldman D, Thompson C, Mac-Thiong JM. Prediction of functional recovery six months following traumatic spinal cord injury during acute care hospitalization. J Spinal Cord Med. 2017:1-9 https://doi.org/10.1080/10790268.2017.1279818.

5. Fehlings MG, Vaccaro A, Wilson JR, Singh A, W Cadotte D, Harrop JS. et al. Early versus delayed decompression for traumatic cervical spinal cord injury: results of the surgical timing in acute spinal cord injury study (STASCIS). PLoS ONE. 2012;7:e32037

6. Fehlings MG, Tetreault LA, Wilson JR, Aarabi B, Anderson P, Arnold PM, et al. A Clinical Practice Guideline for the Management of Patients With Acute Spinal Cord Injury and Central Cord Syndrome: recommendations on the Timing $(\leq 24 \mathrm{~h}$ Versus $>24$ h) of Decompressive Surgery. Glob Spine J. 2017;7:195S-202S.

7. Burke JF, Yue JK, Ngwenya LB, Winkler EA, Talbott J, Pan J, et al. 182 Ultra-early $(<12 \mathrm{~h})$ decompression improves recovery after spinal cord injury compared to early $(12-24 \mathrm{~h})$ decompression. Neurosurgery. 2016;63:172.

8. Bourassa-Moreau E, Mac-Thiong JM, Li A, Ehrmann Feldman D, Gagnon DH, Thompson C, et al. Do patients with complete spinal cord injury benefit from early surgical decompression? Analysis of neurological improvement in a prospective cohort study. J Neurotrauma. 2016;33:301-6.

9. Thompson C, Feldman DE, Mac-Thiong JM. Surgical management of patients following traumatic spinal cord injury: identifying barriers to early surgery in a specialized spinal cord injury center. J Spinal Cord Med. 2016;41:1-7.

10. Kirshblum SC, Burns SP, Biering-Sorensen F, Donovan W, Graves DE, Jha A, et al. International standards for neurological classification of spinal cord injury (Revised 2011). J Spinal Cord Med. 2011;34:535-546.

11. Park JH, Kim JH, Roh SW, Rhim SC, Jeon SR. Prognostic factor analysis after surgical decompression and stabilization for cervical spinal-cord injury. Br J Neurosurg. 2017;31:194-8.

12. Breiman L, Friedman J, Stone CJ, Olshen RA. Classification and regression trees: CRC press; Boca Raton, FL, 1984.

13. Mirza SK, Krengel WFI, Chapman JR, Anderson PA, Bailey JC, Grady MS, et al. Early versus delayed surgery for acute cervical spinal cord injury. Clin Orthop Relat Res. 1999;359:104-114. 
14. Papadopoulos SM, Selden NR, Quint DJ, Patel N, Gillespie B, Grube S. Immediate spinal cord decompression for cervical spinal cord injury: feasibility and outcome. J Trauma. 2002;52:323-332.

15. Umerani MS, Abbas A, Sharif S. Clinical outcome in patients with early versus delayed decompression in cervical spine trauma. Asian Spine J. 2014;8:427-434.

16. Scivoletto G, Tamburella F, Laurenza L, Molinari M. The spinal cord independence measure: how much change is clinically significant for spinal cord injury subjects. Disabil Rehabil. 2013;35:1808-13.

17. Scivoletto G, Tamburella F, Laurenza L, Molinari M. Distribution-based estimates of clinically significant changes in the International Standards for Neurological Classification of Spinal Cord Injury motor and sensory scores. Eur J Phys Rehab Med. 2013;49:373-384.

18. Grassner L, Wutte C, Klein B, Mach O, Riesner S, Panzer S, et al. Early decompression $(<8 \mathrm{~h})$ after traumatic cervical spinal cord injury improves functional outcome as assessed by spinal cord independence measure after one year. J Neurotrauma. 2016;33:1658-66.

19. El Tecle NE, Dahdaleh NS, Hitchon PW. Timing of surgery in spinal cord injury. Spine. 2016;41:E995-e1004.

20. Batchelor PE, Wills TE, Skeers P, Battistuzzo CR, Macleod MR, Howells DW, et al. Meta-analysis of pre-clinical studies of early decompression in acute spinal cord injury: a battle of time and pressure. PLoS ONE. 2013;8:e72659.

21. Lim JH, Jung CS, Byeon YE, Kim WH, Yoon JH, Kang KS, et al. Establishment of a canine spinal cord injury model induced by epidural balloon compression. J Vet Sci. 2007;8:89-94.
22. Rahimi-Movaghar V, Yazdi A, Karimi M, Mohammadi M, Firouzi M, Zanjani LO, et al. Effect of decompression on complete spinal cord injury in rats. Int J Neurosci. 2008;118:1359-73.

23. Batchelor PE, Kerr NF, Gatt AM, Aleksoska E, Cox SF, GhasemZadeh A, et al. Hypothermia prior to decompression: buying time for treatment of acute spinal cord injury. J Neurotrauma. 2010;27:1357-68.

24. Shields CB, Zhang YP, Shields LB, Han Y, Burke DA, Mayer $\mathrm{NW}$. The therapeutic window for spinal cord decompression in a rat spinal cord injury model. J Neurosurg Spine. 2005;3:302-7.

25. Piazza M, Schuster J. Timing of surgery after spinal cord injury. Neurosurg Clin N Am. 2017;28:31-39.

26. Yousefifard M, Rahimi-Movaghar V, Baikpour M, Ghelichkhani $\mathrm{P}$, Hosseini M, Jafari A, et al. Early versus late spinal decompression surgery in treatment of traumatic spinal cord injuries; a systematic review and meta-analysis. Emerg (Tehran). 2017;5:e37.

27. Marino RJ, Ditunno JF Jr, Donovan WH, Maynard F Jr.. Neurologic recovery after traumatic spinal cord injury: data from the Model Spinal Cord Injury Systems. Arch Phys Med Rehabil. 1999;80:1391-96.

28. Fawcett JW, Curt A, Steeves JD, Coleman WP, Tuszynski MH, Lammertse D, et al. Guidelines for the conduct of clinical trials for spinal cord injury as developed by the ICCP panel: spontaneous recovery after spinal cord injury and statistical power needed for therapeutic clinical trials. Spinal Cord. 2007;45:190-205.

29. Kirshblum SC, O'Connor KC. Predicting neurologic recovery in traumatic cervical spinal cord injury. Arch Phys Med Rehabil. 1998;79:1456-66.

30. Shrier I, Platt RW. Reducing bias through directed acyclic graphs. BMC Med Res Methodol. 2008;8:70. 